\title{
Preparation and thermal stability of nickel nanowires via self-assembly process under magnetic field
}

\author{
HU WANG, MING LI, XIAOYU LI, KENAN XIE and LI LIAO* \\ School of Chemical Engineering, Sichuan University, Chengdu 610065, People's Republic of China
}

MS received 26 January 2015; accepted 7 May 2015

\begin{abstract}
Nickel nanowires were synthesized via a template-free method in an aqueous solution system combined with chemical reduction and magnetic field. The suitable concentration of $\mathrm{Ni}$ ions and reaction time were controlled in order to obtain nickel wires with uniform sizes. The products were characterized by $X$-ray diffraction, scanning electron microscopy, transmission electron microscopy, thermogravimetry and differential scanning calorimetry. The results showed that the Ni nanowires with large aspect ratio up to 200 had uniform size and morphology, about $200 \mathrm{~nm}$. Especially, it is noteworthy that the samples were stable in air when the temperature was lower than $318^{\circ} \mathrm{C}$. The study would provide a facile method to prepare nickel nanowires with homogeneous diameter and high thermal stability, which could be used in catalysing $\mathrm{CO}_{2}$ hydrogenation.
\end{abstract}

Keywords. Nickel nanowires; magnetic field; self-assembly; thermal stability.

\section{Introduction}

In recent years, catalytic hydrogenation of $\mathrm{CO}_{2}$ to light hydrocarbon has been recognized as one of the most effective and economical ways to fix and utilize a large amount of anthropogenic $\mathrm{CO}_{2}$ and plays a key role in the energy field. ${ }^{1-3}$ Nickel is considered as efficient hydrogenation catalyst both in the conversion and selectivity of $\mathrm{CO}_{2}$ hydrogenation, which has been extensively applied in industry. ${ }^{4}$ Nanoparticles of metals possess the advantages of small grain diameter and high-specific surface area ${ }^{5}$ however, the active region on the surface cannot be exposed because of aggregation of the particles. ${ }^{6,7}$ In contrast, one-dimensional nanostructures especially nanowires of metallic materials have much advantages of more active region and more abundant interspaces owing to their unique morphology with a space grid structure. Therefore, it is of great significance to prepare nickel nanowires by an effective method.

Numerous synthesis methods of iron group nanowires have been developed to prepare nanoscale magnetic metal materials. ${ }^{8}$ The approaches of nanowires preparation can be divided into two broad categories: vapour-phase techniques and liquid- or solution-based techniques. The vapour-phase techniques mainly include methods such as chemical vapour deposition (CVD) using catalyst metals, reactive vapour transport, carbothermal reduction, thermal evapouration and thermal decomposition but it remains the disadvantages of high cost and complicated

*Author for correspondence (liaolis@ @ scu.edu.cn) equipments. Liquid- or solution-based techniques have great industrial application prospect, which include approaches such as sol-gel synthesis, hydrothermal processes and electrodeposition. ${ }^{9}$ Many liquid-phase techniques utilize templates for producing one-dimensional materials. The methods with templates have notable advantages, from which highly ordered and size-controlled nanowires can be obtained. ${ }^{10}$ However, the template methods require several steps, including fabrication and removal of templates in order to obtain bare nanowires, which hinders its large-scale applications. ${ }^{11-13}$

Therefore, it is of great significance and necessity to develop a practical approach to fabricate nanowires, which has the advantages over other methods and have lower temperature, lower cost, less hazardous, simpler equipments, higher yield and more uniform nanowires. ${ }^{14}$ Much attention had been paid to the effect of a magnetic field on the nucleation and growth process of magnetic materials and on the self-assembly behaviour of magnetic nanocrystals. ${ }^{15-17}$ It has been indeed found that the magnetic field can significantly influence the movement of magnetic particles. ${ }^{18}$ It is, therefore, significant to study the growth and self-assembly behaviour of magnetic nanocrystals under an external magnetic field.

In this paper, a template-free method by combining chemical reduction and magnetic field was applied to prepare nickel nanowires. The properties of prepared nickel nanowires were investigated. Nickel particles were prepared in the absence of a magnetic field to better illustrate the structure directing role of the magnetic field. In order to control the morphology of nickel nanowires, the growth behaviour of nickel was studied. It is possible that 
the reduction rate of $\mathrm{Ni}(\mathrm{II})$ ions affects the morphology of nickel nanowires.

\section{Experimental}

\subsection{Materials and methods}

In the experiments, all the reagents were analytical grade and used as received. The reaction solutions were prepared using nickel sulphate hexahydrate $\left(\mathrm{NiSO}_{4} \cdot 6 \mathrm{H}_{2} \mathrm{O}\right)$ as a source of $\mathrm{Ni}(\mathrm{II})$ ions, deionized water as a solvent, hydrazine monohydrate $\left(\mathrm{N}_{2} \mathrm{H}_{4} \cdot \mathrm{H}_{2} \mathrm{O}\right)$ as a reducing agent and sodium hypophosphite monohydrate $\left(\mathrm{NaH}_{2} \mathrm{PO}_{2} \cdot \mathrm{H}_{2} \mathrm{O}\right)$ as an initiator. Sodium hydroxide $(\mathrm{NaOH})$ was added as a source of $\mathrm{OH}^{-}$ions. Trisodium citrate dihydrate $\left(\mathrm{C}_{6} \mathrm{H}_{5} \mathrm{Na}_{3} \mathrm{O}_{7} \cdot 2 \mathrm{H}_{2} \mathrm{O}\right)$ was used as a complexing agent and a nucleating agent.

For the synthesis of nickel wires, the reaction solution was kept at $65^{\circ} \mathrm{C}$ in a water bath, where two parallel neodymium magnets $(60 \times 30 \mathrm{~mm})$ were located inside. The magnetic field intensity was about $15 \mathrm{mT}$, which is measured by a Tesla meter. In a typical experiment, $0.1 \mathrm{~g}$ $\mathrm{NiSO}_{4} \cdot 6 \mathrm{H}_{2} \mathrm{O}, 0.12 \mathrm{~g} \mathrm{C}_{6} \mathrm{H}_{5} \mathrm{Na}_{3} \mathrm{O}_{7} \cdot 2 \mathrm{H}_{2} \mathrm{O}$ and $60 \mathrm{ml}$ deionized water were mixed in a teflon beaker. A grass-green colour transparent solution was observed after strongly stirring for half an hour. Then $0.25 \mathrm{~g} \mathrm{NaOH}$ was added in the solution after the mixture was heated to $65^{\circ} \mathrm{C}$. After that $2 \mathrm{ml}$ hydrazine monohydrate solution $(80 \mathrm{wt} \%)$ and $0.01 \mathrm{~g} \mathrm{NaH}_{2} \mathrm{PO}_{2} \cdot \mathrm{H}_{2} \mathrm{O}$ were added into the solution, and the colour changed from grass green to sky blue. After several minutes, nickel wires were washed three times with deionized water and ethanol. The final products were dispersed in ethanol or dried in a vacuum oven at $50^{\circ} \mathrm{C}$ for $24 \mathrm{~h}$.

\subsection{Characterization}

The size and morphology of the products were characterized on a field-emission scanning electron microscope (FESEM, JHOL, S-450). The composition and crystallographic properties of the products were analysed by $\mathrm{X}$-ray diffraction (XRD, Philips, X'pert) in the range from $20^{\circ}$ to $90^{\circ}$. Transmission electron microscopy (TEM), high-resolution TEM (HRTEM) images and selected area electron diffraction (SAED) patterns were obtained by a JEOL JEM-100CX TEM. The Ni samples were heated from 30 to $800^{\circ} \mathrm{C}$ at a rate of $10^{\circ} \mathrm{C} \mathrm{min}{ }^{-1}$ by a thermogravimetric (TG) analyser and differential scanning calorimeter (DSC) (NETZSCH STA 449C) in air atmosphere to study its thermostability.

\section{Results and discussion}

\subsection{Self-assembly process and preparation of nickel nanowires}

Figure 1 shows the SEM images of nickel particles and wires synthesized from the solutions under a magnetic field at different times.
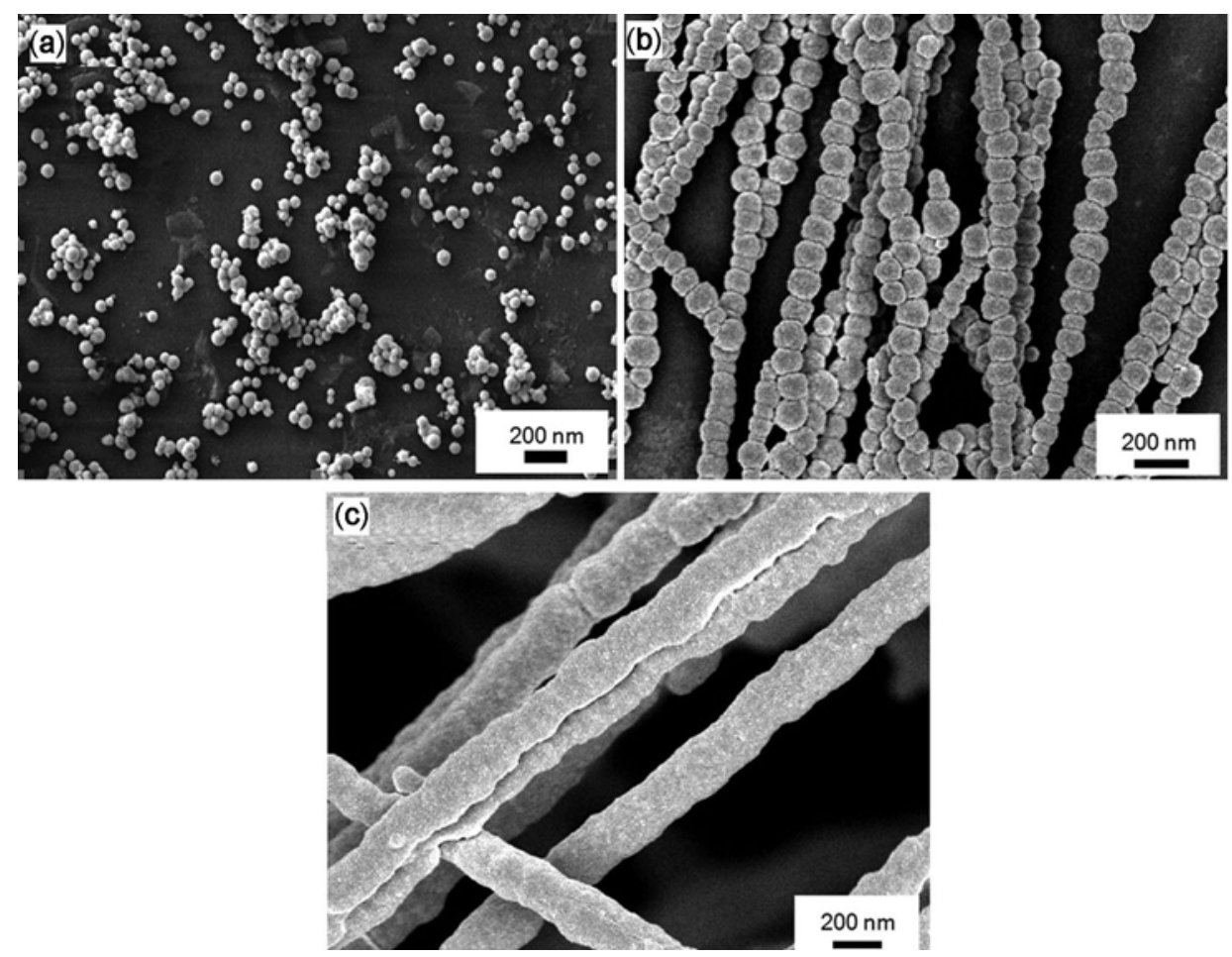

Figure 1. SEM micrographs of products prepared after reaction proceeded (a) $10 \mathrm{~min}$, (b) $20 \mathrm{~min}$ and (c) $30 \mathrm{~min}$. 
Figure 1a shows the surface morphology of nanoparticles prepared after a tiny amount of initiators were added in the solution $10 \mathrm{~min}$ later. Only single spherical nanoparticle with an average particle size of $80 \mathrm{~nm}$ can be observed, which can be inferred that the external magnetic field did not play an obvious directing role on the formation of the nanoparticles when the particle size was less than a critical value.

Figure 1b shows the nickel nanochains after the reaction was proceeded $20 \mathrm{~min}$. Single nanoparticles began to be arranged along a straight line under the magnetic field and single dispersed particles were disappeared in the visual field. The average particle size was about $150 \mathrm{~nm}$, which illustrated the fact that the particle diameter was continuously increased when the reaction proceeded.

Figure 1c shows the SEM photograph of nickel nanowires prepared after reacting for $30 \mathrm{~min}$, and the linear nanowires were formed instead of bead chain. Nickel ions in the solution were then reduced on the surface of the prepared nickel nanochains due to the autocatalysis of nickel, which led to the increase of diameter and better linearity of nickel nanowires. And the surface of prepared nickel nanowires was relatively smoother than nickel nanochains.

Figure 2 presents the difference in morphology between the eventual products prepared with (a) and without (b) an external magnetic field applied during particle's growth. The morphology of (b) appears disorderly, consisting of single nearly spherical nanoparticles, while (a) contains highly oriented straight wires; it indicates that a magnetic field can effectively affect the aggregation of nickel nanocrystals. When no external magnetic field is applied, the orientation of each magnetic domain is spontaneously random. The nanocrystals may magnetize one another by dipolar interaction in an arbitrary direction, which cannot finally result in the formation of nanowires. The XRD patterns of the products prepared with and without an external magnetic field applied are similar, which suggested that the external magnetic field affect the morphology of nickel nanowires merely.

To verify the formation mechanism of nickel wires under an external magnetic field various parallel experiments were carried out. The regular patterns can be inferred that the length and diameter had an increasing trend with the extension of reaction time, as shown in figure 3 . When the reaction proceeded slowly, spherical particles would have enough time to grow in a larger size. As a result, the growth trend along the direction of magnetic field was restrained in some degree, which led to a short-length and chain-like nickel wires. On the other hand, it may be tough to control the morphology to be linear and uniform with an excessively fast reaction rate. However, the actual aggregating process is very complicated, determined by nucleation, spacial hindrance, magnetic attraction of excessive finer particles and other kinetic factors. When the reaction rate was appropriately
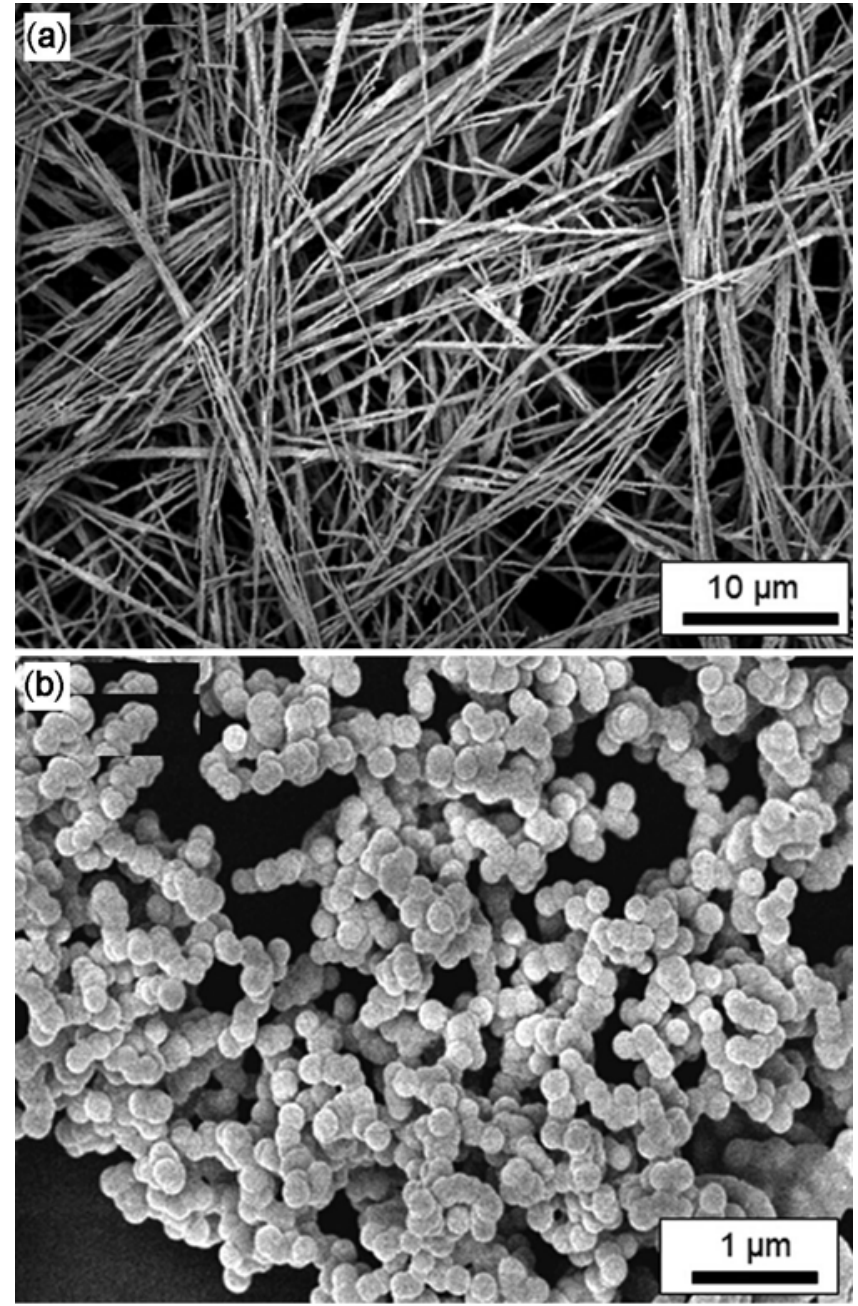

Figure 2. SEM micrographs of products prepared (a) with and (b) without an external magnetic field.

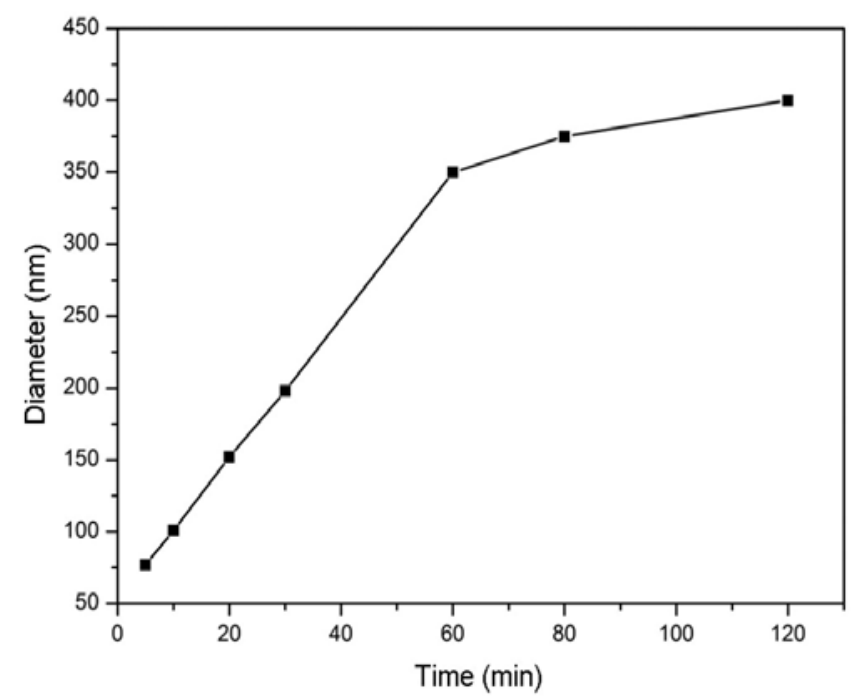

Figure 3. Relationship of the reaction time and particle diameter. 
controlled, acicular nickel nanowires with diameter from 200 to $500 \mathrm{~nm}$ can be prepared.

\subsection{XRD, TEM with SAED of nickel nanowires}

Figure 4 shows the corresponding XRD spectrum for the resultant wires. It can be well indexed with the reflections of face-centred cubic Ni (PDF standard cards, JCPDS 040850, space group Fm-3m), without impurity peaks. Three characteristic peaks $\left(2 \theta=44.46^{\circ}, 51.81^{\circ}\right.$ and $\left.76.49^{\circ}\right)$ corresponding to Miller indices (111), (200) and (220), respectively, were observed. The cell parameter was $a=3.524 \mathrm{~nm}$, $b=3.524 \mathrm{~nm}$ and $c=3.524 \mathrm{~nm}$. The average crystalline grain size of linear powders was $20.7 \mathrm{~nm}$, calculated from the XRD patterns according to the Scherrer formula: $L=\lambda k / \cos \beta \theta$. It was obviously smaller than the size of the particles in FESEM micrograph, which implied that one particle was consisted of several crystalline grains.

Figure 5 exhibits typical TEM image of the nickel nanowires and the SAED pattern. It can be seen that nickel particles are assembled well to solid linear structure under the magnetic field. The SAED pattern is composed of several diffraction rings, indicating the polycrystalline property, and the corresponding crystal face is marked on the photograph.

\subsection{Thermal stability of nickel nanowires}

It is essential to study the stability of the nickel nanowires in air atmosphere considering its application in hydrogenation. The prepared nanowires were characterized by using TG analyser and DSC. As shown in figure 6, between 30 and $318.0^{\circ} \mathrm{C}$, there was a little weight loss which may be caused by the evapouration of remained liquid in the samples, nickel nanowires began to oxidize

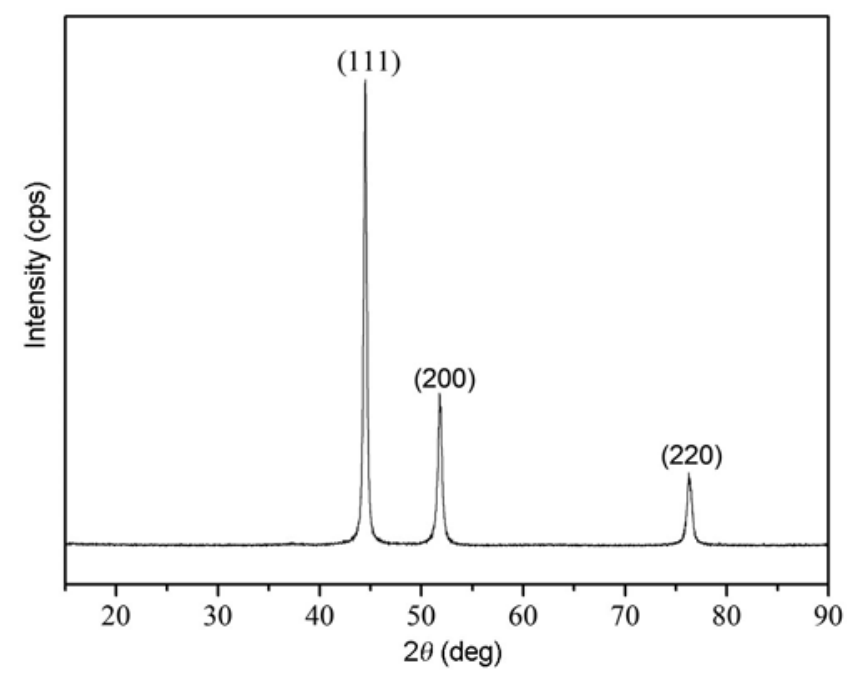

Figure 4. XRD pattern of nickel nanowires. at $318.0^{\circ} \mathrm{C}$ and the oxidation rate approaches to maximum at $447.4^{\circ} \mathrm{C}$. The final weight gain is about $22.55 \%$ which is different with the theoretical weight gain $(27.3 \%)$ for perfect conversion of pure $\mathrm{Ni}$ to $\mathrm{NiO}$. This datum indicates that the oxidation process from $\mathrm{Ni}$ to $\mathrm{NiO}$ is not complete yet at about $900^{\circ} \mathrm{C}$. The DSC curve declines and an exothermic peak appears at $882.1^{\circ} \mathrm{C}$, it may be caused by the crystal transformation of nickel at $882.1^{\circ} \mathrm{C}$. And it can be inferred that the nickel wires fabricated via the liquid phase method without template are thermally stable in air below $318^{\circ} \mathrm{C}$, which would largely expand its application.

To further analyse the stability of nickel nanowires under air atmosphere, nickel nanowires were annealed in air at various temperatures. Figure 7 shows XRD patterns of nickel nanowires annealed at different temperatures. Compared with XRD pattern at room temperature, characteristic peaks of $\mathrm{NiO}$ appeared at $300^{\circ} \mathrm{C}$, which illustrated that the sample had been oxidized. When the annealing temperature rose to $500^{\circ} \mathrm{C}, \mathrm{NiO}$ occupied a

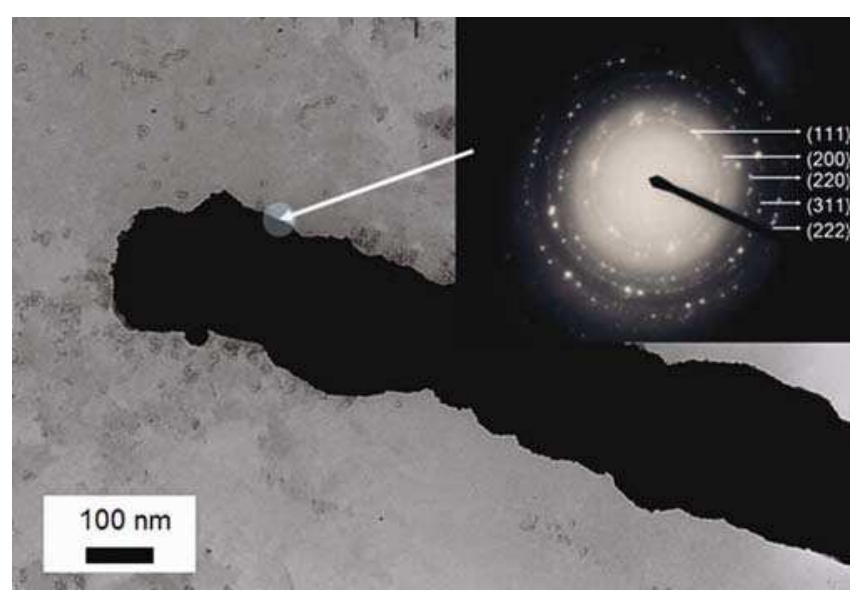

Figure 5. TEM image of nickel nanowires with selected area electron diffraction (SAED) pattern.

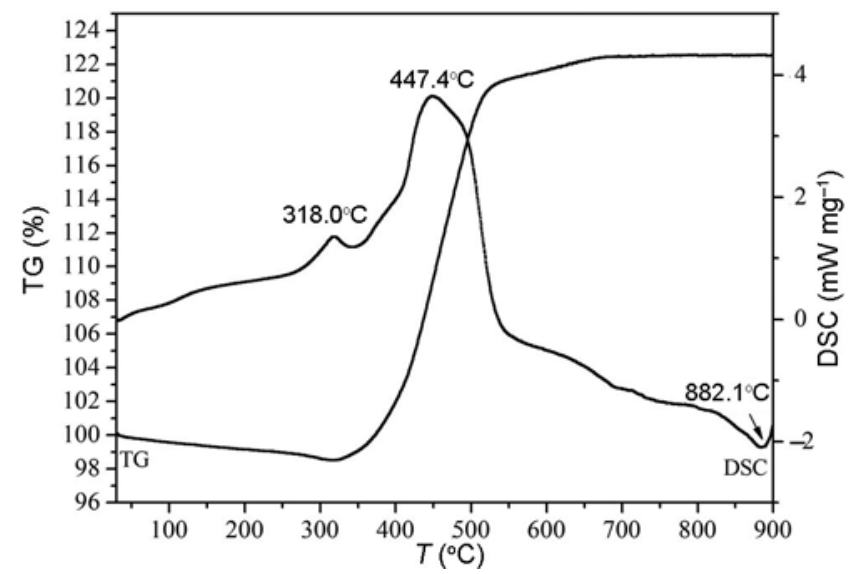

Figure 6. TG/DSC analysis of nickel nanowires. 


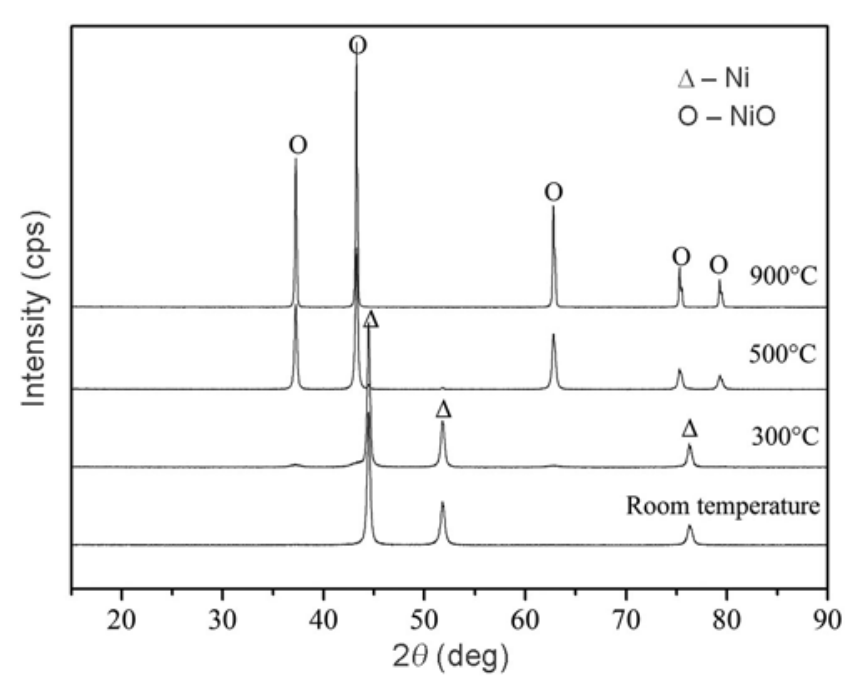

Figure 7. XRD patterns of prepared nickel nanowires annealed in air atmosphere at various temperatures.

strong majority in the annealed samples; meanwhile, hardly the characteristic peaks of $\mathrm{Ni}$ can be found. As the annealing temperature rose to $900^{\circ} \mathrm{C}, \mathrm{Ni}$ was totally oxidized to $\mathrm{NiO}$. However, it cannot be easily jumped to the conclusion that there is no transformation to $\mathrm{NiO}$ in air atmosphere above $500^{\circ} \mathrm{C}$ just on account of the similar $\mathrm{XRD}$ patterns from 500 to $900^{\circ} \mathrm{C}$. An interesting phenomenon was discovered that the colour was diverse at different annealing temperatures, which indicated the existence of chemical transformation in the process. Light grey, bottle green colour appeared, corresponding to 500 and $900^{\circ} \mathrm{C}$, respectively. Cubic $\mathrm{NiO}$ (PDF standard cards, JCPDS 47-1049, space group Fm-3m) and hexagonal $\mathrm{NiO}$ (PDF standard cards, JCPDS 44-1159, space group $\mathrm{R}-3 \mathrm{~m}$ ) coexisted from 500 to $900^{\circ} \mathrm{C}$ and the characteristic peaks of them was too close to be distinguished. Hexagonal $\mathrm{NiO}$ decreases with the rising of temperature and cubic $\mathrm{NiO}$ increases, from which it can be inferred that cubic $\mathrm{NiO}$ is more stable and appears green. To sum up, the colour change of $\mathrm{NiO}$ may be caused by the crystal transformation.

\section{Conclusions}

Polycrystal nickel nanowires with an average diameter of about $200 \mathrm{~nm}$ were successfully prepared by a templatefree method combined chemical reduction and magnetic field. It is found that an external magnetic field can make acicular nickel wires one-dimensionally self-assembled with their magnetic easy axes aligned along the magnetic line of force, leading to the formation of polycrystal nanowires. Nickel particles aggregated larger spherical particles rather than nickel nanowires in the absence of a magnetic field. In the present work, it can be inferred that the rate of reaction played a crucial role on the morphology of nickel nanowires. When the reaction proceeded excessively slow or rapid, acicular nickel nanowires with relatively low diameter and long length can hardly be obtained. Nickel nanowires are stable in air when the temperature is lower than $318^{\circ} \mathrm{C}$. The present study provides a facile method to prepare nickel nanowires with homogeneous diameter in a large scale, which would broad their practical applications.

\section{Acknowledgements}

Financial supports by the National Natural Science Foundation of China (Grant no. 50904046) are gratefully acknowledged.

\section{References}

1. Janke C, Duyar M S, Hoskins M and Farrauto R 2014 Appl. Catal. B: Environ. 152-153 184

2. Tao X M, Wang J M, Li Z W and Ye Q G 2013 Comput. Theor. Chem. $\mathbf{5 9} 1023$

3. Satthawong R, Koizumi N, Song C S and Prasassarakich P 2013 J. $\mathrm{CO}_{2}$ Utiliz. 3102

4. Tang S C, Zheng Z, Vongehr S and Meng X K 2011 J. Nanopart. Res. 137085

5. Karim S and Maaz K 2011 Mater. Chem. Phys. 1301103

6. Zhang H J and Liu Y 2008 J. Alloys Compd. 458588

7. Lin S W, Chang S C, Liu R S, Hu S F and Jan N T 2004 J. Magn. Magn. Mater. 28228

8. Chen D L and Gao L 2005 Chem. Phys. Lett. 405 159

9. Han S, Chen H Y, Chen C C and Yuan T N 2007 Mater. Lett. 611105

10. Garcia C, Lecante P, Neumeyer D and Verelst M 2008 Mater. Lett. 622106

11. Rahman Z, Razeeb M, Rahman A and Kamruzzaman D 2003 J. Magn. Magn. Mater. 262166

12. Huang D, Zhang Q and Qiao P Z 2011 Comput. Mater. Sci. 50903

13. Byrne F, Mello P, Whelan A and Davies A 2009 J. Magn. Magn. Mater. 3211341

14. Shi J B, Chen Y C, Lee C W, Wu C and Chen C J 2008 Mater. Lett. 622218

15. Peng C X, Gong J H and Wang L 2009 Comput. Mater. Sci. 16229

16. Hellenthal C, Ahmed W, Kooij S and Silfhout V 2012 J. Nanopart. Res. 141107

17. Moustafa F and Daoush M 2007 J. Mater. Process. Technol. 18159

18. Chen H Y, Xu C J, Zhou X, Liu Y Q and Zhao G Z 2012 Mater. Res. Bull. 474353 